\title{
Mediastinal Adenopathy in Lung Cancer- Relative Merits of 18F-FDG PET-CT and Different Imaging Modalities-A Short Review
}

\author{
${ }^{1}$ Hafizur Rahman and ${ }^{2}$ Shamim M F Begum \\ ${ }^{1}$ Institute of Nuclear Medicine \& Allied sciences, Faridpur \\ ${ }^{2}$ National Institute of Nuclear Medicine and Allied Sciences
}

Correspondence Address : Dr. Hafizur Rahman, Principle Medical Officer, Institute of Nuclear Medicine \& Allied Sciences, Faridpur, E-mail: mhrn08@yahoo.com

Lung cancer is a leading cause of death from cancer among men worldwide. In United States, 5-years survival rate of lung cancer is only $15 \%$. In Bangladesh lung cancer is the commonest male cancer and accounts for $28.39 \%$ of all cancer patients $(1,2)$. The two main histo-pathologic categories of lung cancer are non-small cell lung cancer (NSCLC) or small cell lung cancer (SCLC). The majority of them is NSCLC, comprises $87 \%$ of all lung cancers. Metastatic involvement of mediastinal lymph nodes is a very common phenomenon in lung cancer. The status of regional lymph node is an important prognostic factor. Prognosis is also largely depended on the early diagnosis and precise staging of the disease. The treatment planning depends on tumor stage (3).

TNM staging system is the standard tool for staging of lung cancer. This staging system is maintained and kept up-to-date by the American Joint Committee on Cancer and the International Union Against Cancer. TNM staging is based on a combination of findings: the location and extent of the primary tumor (T), the presence or absence of intrapulmonary, hilar or mediastinal lymph node metastases $(\mathrm{N})$, and the presence or absence of extrathoracic metastases (M). The combination of $\mathrm{T}, \mathrm{N}$, and $\mathrm{M}$ staging is used to give the tumor an overall stage I to stage IV, thereby grouped the patients into similar prognostic stages (4).

The International Association for the Study of Lung
Cancer (IASLC) serves as the primary source of recommendations for lung cancer staging revisions and IASLC is recognized by the International Union Against Cancer (UICC). For lung cancer staging 7th edition of the TNM staging is the latest version and incorporates several proposed revisions to better align. It has been validated on the basis of evidences from larger world wide database $(4,5)$.

Here we discuss about the common pitfalls of mediastinal adenopathy in lung cancer and relative merits of 2-(fluorine -18) fluoro-2 deoxy-D-glucose (18F FDG) positron emission tomography (PET), magnetic resonance imaging (MRI) imaging and computed tomography (CT).

The conventional methods for mediastinal staging include computed tomography (CT), magnetic resonance imaging (MRI) and mediastinoscopy with various biopsy procedures. Compared to the invasive procedures the noninvasive imaging modalities CT and MRI are lack of sensitivity and specificity for accurate meditational nodal staging in patients with lung cancer. However, biopsy procedures are inconvenient and potentially risky $(6,7)$.

CT is the most widely available and commonly used imaging technique to assess intra- and extra-thoracic metastases. However, CT has been shown to have limited abilities when used as the sole modality in evaluating the mediastinum for metastases. Evaluation on conventional CT imaging is based upon size. 
Lymph nodes with a short axis diameter of greater than $1.0 \mathrm{~cm}$ are suspected of representing metastatic disease. Unfortunately, up to $40 \%$ of nodes larger than $1 \mathrm{~cm}$ is benign, which are might be due to inflammatory process, simple hyperplasia or reactive etiology. Other specific challenges include the presence of metastatic disease in normal size nodes $(<1 \mathrm{~cm}$ in the short axis), difficulty in identifying disease in certain nodal stations, subaortic or window lymph nodes or in the presence of obstructive pneumonitis or atelectasis $(7,8)$.

The role of MRI in the mediastinal staging in lung cancer is not as well studied as CT. Studies showed only few exceptions MRI offered no advantages when compared with CT. It has the same disadvantages of the size-related definition of lymph node positivity as CT, which needs to use of contrast-enhanced MRI for improvement of staging $(9,10)$. MRI can assess the hilar and aortopulmonary nodes more accurately than $\mathrm{CT}$, because of its better distinction between lymph nodes and blood vessels. Comparing CT and MRI, Webb et al reported that MRI has got a sensitivity of $64 \%$, a specificity of $48 \%$, and an accuracy of $61 \%$ (11). Patterson et al reported better operating characteristics for MRI with a sensitivity $71 \%$, specificity $91 \%$, and accuracy $83 \%$ (12).

18F FDG PET is a potential tool of staging in patients with NSCLC. PET imaging can contribute complementary non-invasive functional information to the established size criteria for abnormal mediastinal lymph nodes, which improve the overall accuracy of clinical staging for lung cancer. FDG-PET is superior to $\mathrm{CT}$ and MRI in staging of metastatic mediastinal lymph nodes. FDG uptake within a normal sized node is highly suggestive of metastatic disease and should be considered to represent malignancy until proven otherwise. The absence of FDG uptake in enlarged nodes identified at CT is highly indicative of lack of metastatic involvement.
Dwamena et al performed a meta-analysis of 14 PET studies for staging mediastinal adenopathy and found an overall sensitivity of $79 \%$ and an overall specificity of $91 \%$. Accuracy was $92 \%$, positive predictive value was $90 \%$ and negative predictive value was $93 \%$. This is clearly superior to $\mathrm{CT}$ which has an overall reported sensitivity and specificity of $62 \%$ and $73 \%$, respectively. PET staging of mediastinal nodes approaches mediastinoscopy, which has reported sensitivity between $72 \%$ to $94 \%$. Results of the PET exam can also be used to more accurately guide mediastinal exploration with improve yield from preoperative mediastinal lymph node sampling (9).

The PET-CT is an integral part in quality management of lung cancer patients in developed countries. By integrating functional and anatomic data, PET-CT improves the $\mathrm{N}$ staging compared with PET or CT alone. Studies demonstrated increased sensitivity, specificity, positive predictive value, negative predictive value, and accuracy of PET-CT for detecting metastatic lymph nodes of, respectively, $73 \%, 91 \%, 71 \%, 90 \%$, and $86 \%$ versus $83 \%, 81 \%$, $71 \%, 89 \%, 82 \%$ of PET alone and $74 \%, 73 \%, 52 \%$, $88 \%, 73 \%$ of CT alone $(10,11)$. The improved sensitivity of PET-CT may leads to greater number of false positive cases and reduced specificity. The number of false positive exams can be decreased by applying criteria regarding the attenuation density of the lymph node into image analysis. Lymph nodes with tracer uptake greater than mediastinal blood pool activity, but showing high attenuation on CT are considered by some authors to be benign.

Due to high negative predictive value PET-CT can reduce invasive meditational staging or mediastinoscopy. PET-CT is the best noninvasive method for the detection of nodal metastasis, but mediastinoscopy remains the gold standard. Compared with mediastinoscopy and surgical staging, the reported accuracy of PET and PET/CT in lymph node staging is $56 \%$ and $78 \%$, respectively $(10,12)$. 
Clinicians must understand the details set forth in the TNM classification system and be familiar with the changes in the 7 th edition, which attempts to better correlate disease with prognostic value and treatment strategy. By recognizing the relevant radiologic appearances of staging of the disease with the TNM classification system, and being familiar with potential imaging pitfalls, nuclear radiologist or medicine physician can make an important contribution to treatment and outcome in lung cancer patients.

\section{REFERENCES}

1. GLOBOCAN 2002, 2008 Database, International Agency for Research on Cancer, World Health Organization. Available at http://www-dep.iarc.fr/, last accessed January 27, 2010.

2. Hospital Cancer Registry Report 2008-2010 (printed) and 2011-2013, National Institute of Cancer Research and Hospital (NICRH) Dhaka Tribune, Published: 03:12 January 19, 2014.

3. Goldstraw P, Crowley J, Chansky K, et al. The IASLC Lung Cancer Staging Project: Proposals for the Revision of the TNM Stage Grouping in the Forthcoming (seventh) Edition of the TNM Classification of Malignant Tumours. J Thorac Oncol

4. L. L. Carr, J. H. Finigan, and J. A. Kern, Evaluation and Treatment of Patients with Non-Small Cell Lung Cancer. Medical Clinics of North America 2011; 95(6):1041-54.

5. Groome PA, Bolejack V, Crowley JJ, et at. The ISLAC Lung Cancer Staging Project: Validation of the Proposals for Revision of The T,N, and M Descriptors and Consequent Stage Grouping In the Forthcoming (seventh) Edition of the TNM Classification of Malignant Tumours. J Thorac Oncol 2007; 2(8): 694-705.

6. Primack SL, Lee KS, Logan PM, Miller RR, Mu“ller NL. Bronchogenic Carcinoma: Utility of CT in the Evaluation of Patients with Suspected Lesions. Radiology 1994;193:795-800.

7. Mcloud TC, Bourgouin PM, greeerg RW, et al.
Bronchogenic Carcinoma: Analysis of Staging in the Mediastinum with CT by Correlative Lymph node Mapping and Sampling Radiology 1992; 182(2):319-23.

8. Gdeedo A, Van schil P, Corthouts, Van Mieghem F, Van Meerbreeck J, Van Marck E. Prospective Evaluation of Computed Tomography and Mediastinoscopy in Mediastinal Lymph Node Staging . Eur Respir J 1997;10(7):1547-51.

9. Laissy JP, Gay-Depassier P, Soyer P, et al. Enlarged mediastinal lymph nodes in bronchogenic carcinoma: assessment with dynamic contrast-enhanced MR imaging. Work in progress. Radiology. 1994; 191:263-267.

10. Haberkorn U, Schoenberg SO. Imaging of lung cancer with CT, MRT and PET. Lung Cancer 2001;34 (3):13-23.

11. Webb WR, Gatsonis C, Zerhouni EA, et al. CT and MR imaging in staging non-small cell bronchogenic carcinoma: report of the Radiologic Diagnostic Oncology Group. Radiology. 1991;178:705-713.

12. Patterson GA, Ginsberg RJ, Poon PY, et al. A prospective evaluation of magnetic resonance imaging, computed tomography, and mediastinoscopy in the preoperative assessment of mediastinal node status in bronchogenic carcinoma. J Thorac Cardiovasc Surg. 1987;94:679-684.

13. Dwamena BA, Sonnad SS, Angobaldo JO, Walh RL. Metastases from Non-Small Cell Lung Cancer: Mediastinal Staging in the 1990s-Meta-Analytic Comparision of PET and CT. Radiology 1999; 213(2):530-36.

14. G. E. Darling, D. E. Maziak, R. I. Inculet et al. Positron Emission Tomography-Computed Tomography Compared with Invasive Mediastinal Staging in Non-Small Cell Lung Cancer: Results of Mediastinal Staging in the Early Lung Positron Emission Tomography Trial. Journal of Thoracic Oncology 2011;6(8):1367-72.

15. T. Y. Jeon, K. S. Lee, C. A. Yi et al. Incremental Value of PET/CT over CT for Mediastinal Nodal Staging of Nonsmall Cell Lung Cancer: Comparison Between Patients With and Without Idiopathic Pulmonary Fibrosis. American Journal of Roentgenology 2010;195(2):370-76.

16. M. Metin, N. Citak, A. Sayar et al. The Role of Extended Cervical Mediastinoscopy in Staging of Non-small Cell Lung Cancer Of the Left Lung and a Comparison with Integrated Positron Emission Tomography and Computed Tomography: Does Integrated Positron Emission 